\title{
Diagnostics and Treatment of Hepatocellular Carcinoma in 2016: Standards and Developments
}

\author{
Jörg Trojan ${ }^{a, b}$ Stephan Zangos ${ }^{b, c}$ Andreas A. Schnitzbauer ${ }^{b, d}$ \\ ${ }^{a}$ Medizinische Klinik 1, Universitätsklinikum Frankfurt, Frankfurt am Main, Germany; \\ b Universitäres Leberzentrum, Universitätsklinikum Frankfurt, Frankfurt am Main, Germany; \\ cInstitut für Diagnostische und Interventionelle Radiologie, Universitätsklinikum Frankfurt, Frankfurt am Main, Germany; \\ d Klinik für Allgemein- und Viszeralchirurgie, Universitätsklinikum Frankfurt, Frankfurt am Main, Germany
}

\section{Keywords}

Hepatocellular carcinoma - Liver cirrhosis .

Resection - Transplantation - Interventional treatment .

Systemic therapy

\section{Summary}

Background: Hepatocellular carcinoma (HCC) is a frequent complication of liver cirrhosis. Worldwide, HCC is one of the most common cancers, with a rising incidence. Methods: A selective literature search was conducted, taking into account current studies, reviews, meta-analyses, and guidelines. Results: The diagnosis is established either non-invasively by dynamic imaging, showing a typical contrast enhancement and wash-out, or histopathologically. Pathological diagnosis of HCC is recommended for all atypical nodules in patients with cirrhosis and for those in non-cirrhotic patients. Tumor therapy as well as treatment of the underlying chronic liver disease and/or preservation of liver function are important for the management of patients with HCC. Standard stageadapted treatments are based on the widely applied Barcelona Clinic Liver Cancer staging system including liver resection and transplantation, interventional treatments such as thermal ablation and transarterial therapies, and systemic treatment with the tyrosine kinase inhibitor sorafenib. After failure of sorafenib, anti-angiogenic drugs, MET inhibitors, and immunotherapeutics are currently under advanced clinical investigation. Conclusion: Treatment of HCC is multidisciplinary and therefore requires a close cooperation between various disciplines such as hepatology, visceral surgery, radiology, and oncology to achieve the best outcome depending on the tumor stage and degree of liver function impairment.

(C) 2016 S. Karger GmbH, Freiburg

\section{Introduction}

Hepatocellular carcinoma (HCC) is one of the most common tumors worldwide, with more than 30 cases per 100,000 people annually in Southeast Asia and central Africa. Incidence rates of the disease are increasing globally. In the United States, the incidence has doubled over the past 20 years [1]. A similar trend has been observed in Germany [2], with currently around 6,000 new male and 2,500 new female patients per year (fig. 1).

The risk for HCC is influenced by etiology, activity, and stage of underlying liver disease. Patients with liver cirrhosis due to chronic infection with hepatitis $\mathrm{C}$ virus (HCV) or hepatitis B virus (HBV) have the highest risk. The rising incidence in Western countries is mainly due to the still high prevalence of HCV-associated liver cirrhosis and the increasing number of patients with advanced steatohepatitis as hepatic manifestation of the metabolic syndrome (e.g. due to diabetes and obesity) [1]. Several reports indicate that HCC in the setting of metabolic syndrome may also arise in the absence of cirrhosis; however, the exact proportion of this condition or its risk factors are still under investigation [3].

\section{Non-Invasive and Invasive Diagnosis of HCC}

HCC is typically a highly vascular tumor, which can be utilized regarding the non-invasive diagnostics of HCC. Non-invasive diagnosis of HCC in patients with liver cirrhosis is established by one imaging technique (contrast-enhanced computed tomography (CT) or magnetic resonance imaging (MRI)) in nodules larger than $2 \mathrm{~cm}$ showing the HCC radiological hallmark of contrast uptake in the arterial phase and wash-out in the venous/late phase (fig. 2). In nodules between 1 and $2 \mathrm{~cm}$ in size, two coincidental techniques (contrast-enhanced CT, MRI, or ultrasound) are necessary to establish the diagnosis. Pathological diagnosis of HCC is

\section{KARGER}

Fax +497614520714

\section{() 2016 S. Karger GmbH, Freiburg}

$2297-4725 / 16 / 0322-0116 \$ 39.50 / 0$ 
Fig. 1. Annual incidence rates of liver cancer in Germany from 1999 till 2012 for men (blue) and females (red). The numbers given indicate the estimated annual number of patients with newly diagnosed primary liver cancer (ICD-10 code C22) (Zentrum für Krebsregisterdaten im Robert Koch-Institut, www.krebsdaten.de; accessed 10.01.2016).

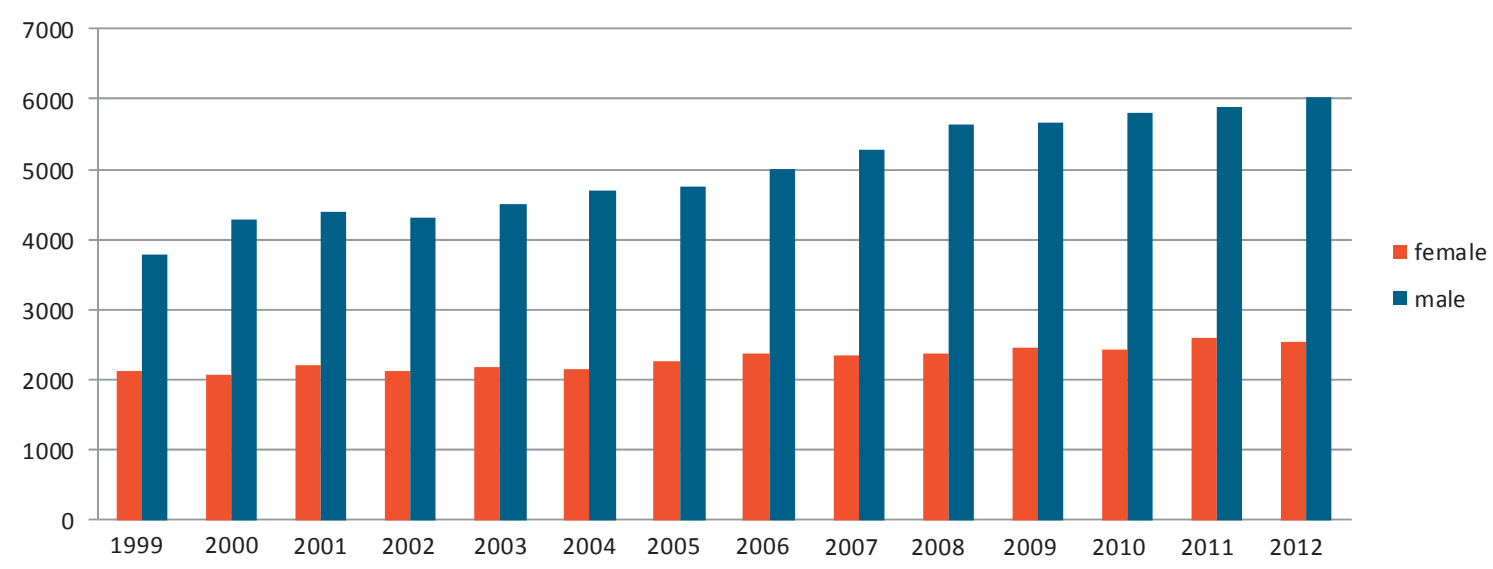

recommended for all nodules occurring in non-cirrhotic livers as well as for those cases with inconclusive or atypical imaging appearance in patients with liver cirrhosis. The histopathological diagnosis is based upon morphological criteria and immunohistochemistry. The marker glypican 3 (GPC3) has a sensitivity of approximately $70 \%$ and a specificity of more than $90 \%$. Further helpful tissue markers are HSP70 and GS. The International Consensus Group of Hepatocellular Neoplasia recommends that a pathological diagnosis of HCC is established if at least two of these markers are positive. Additional staining can be considered to assess neovascularization (CD34) or potential progenitor cell origin (keratin 19, EpCAM) [4]. At the molecular level, HCC is characterized by high-level DNA amplifications in chromosome 6p21 (VEGFA) and 11q13 (FGF19/CNND1) as well as homozygous deletions in chromosome 9 (CDKN2A). The most frequent mutations affect the TERT promoter $(60 \%)$, which is associated with an increased telomerase expression. The TERT promoter can also be affected by copy number variations and hepatitis B DNA insertions; moreover, mutations of it can be found in preneoplastic lesions. TP53 and CTNNB1 are the next most prevalent mutations, affecting $25-30 \%$ of tumors [5]. Some of these changes may cause activation of angiogenesis, which results in the typically seen vascularization of the tumor.

\section{Staging and Therapeutic Algorithm}

HCC includes a wide spectrum of tumors behaving biologically differently. Moreover, allocation of the available treatment options is influenced not only by tumor stage but also by the degree of liver dysfunction. Therefore, accurate staging and classification of the underlying liver disease is crucial for patient management. Several staging systems are well validated and widely used. The Barcelona Clinic Liver Cancer (BCLC) staging system is recommended by the American Association for the Study of Liver Diseases (AASLD) and the European Association for the Study of the Liver (EASL) [4, 6]. Further algorithms are the Hong Kong Liver Cancer (HKLC), the Cancer of the Liver Italian Program (CLIP), the Chinese University Prognostic Index (CUPI), the Japan Integrated Staging (JIS), the Liver Cancer Study Group of Japan (LCSGJ), the Taipei
Integrated Scoring System (TIS), the Okuda, the Chinese University Prognostic Index (CUPI), and the French systems [7].

Using the BCLC algorithm, HCC is categorized as early- (BCLC 0 and BCLC A), intermediate- (BCLC B), advanced- (BCLC C), or end-stage (BCLC D) (fig. 3). Accordingly, surgical (resection or transplantation) or percutaneous thermal therapies (radiofrequency ablation (RFA) or microwave ablation (MWA)) are mainly considered suitable for early stages, while interventional therapies (transarterial chemoembolization (TACE) or radioembolization) are applied in patients with intermediate-stage HCC. Systemic treatment with the tyrosine kinase inhibitor sorafenib is considered as the treatment of choice for patients with advanced-stage HCC. Patients with BCLC stage D do not benefit from cancer treatment, and thus are being considered for best supportive care only.

\section{Liver Resection and Transplantation}

In patients with $\mathrm{HCC}$ without liver cirrhosis, surgical resection is the treatment of choice. In patients with cirrhosis, normal measurements of bilirubin and the absence of portal hypertension (no obvious signs of collaterals, platelets $>100 / \mu \mathrm{l}$, and normal-sized spleen) have been found to be good predictors for a sufficient functional hepatic reserve after resection. Assessing the functional capacity of the remaining liver volume after resection can raise difficulties in the individual case. As a rule, only patients with ChildPugh stage A cirrhosis can undergo resection. Intraoperative ultrasonography has become a standard intraoperative procedure to plan the resection with precision while taking anatomical structures into consideration. A safety margin is desirable, especially in view of existing satellite foci; however, in most cases, only limited resections are possible since the functional hepatic reserve is impaired. The 5-year survival rate after partial liver resection in Western countries is $20-50 \%$. The risk of a tumor recurrence and/or dissemination is highest in the first 3 years postoperatively. In specialized centers, TACE is initially performed in borderline resectable tumors in neoadjuvant intention. However, this approach has not been validated in a randomized fashion yet. Adjuvant treatment after $\mathrm{R} 0$ resection is not established [8]. A complication associated with surgical treatment - in addition to the usual risks as- 
Fig. 2. Modified Barcelona Clinic Liver Cancer (BCLC) staging system. HCC is categorized as early- (BCLC 0 and BCLC A), intermediate- (BCLC B), advanced-(BCLC C), or end-stage (BCLC D) [4]. RFA = Radiofrequency ablation; TACE $=$ transarterial chemoembolization; SIRT = selective intra-arterial radioembolization; $\mathrm{BSC}=$ best supportive care.

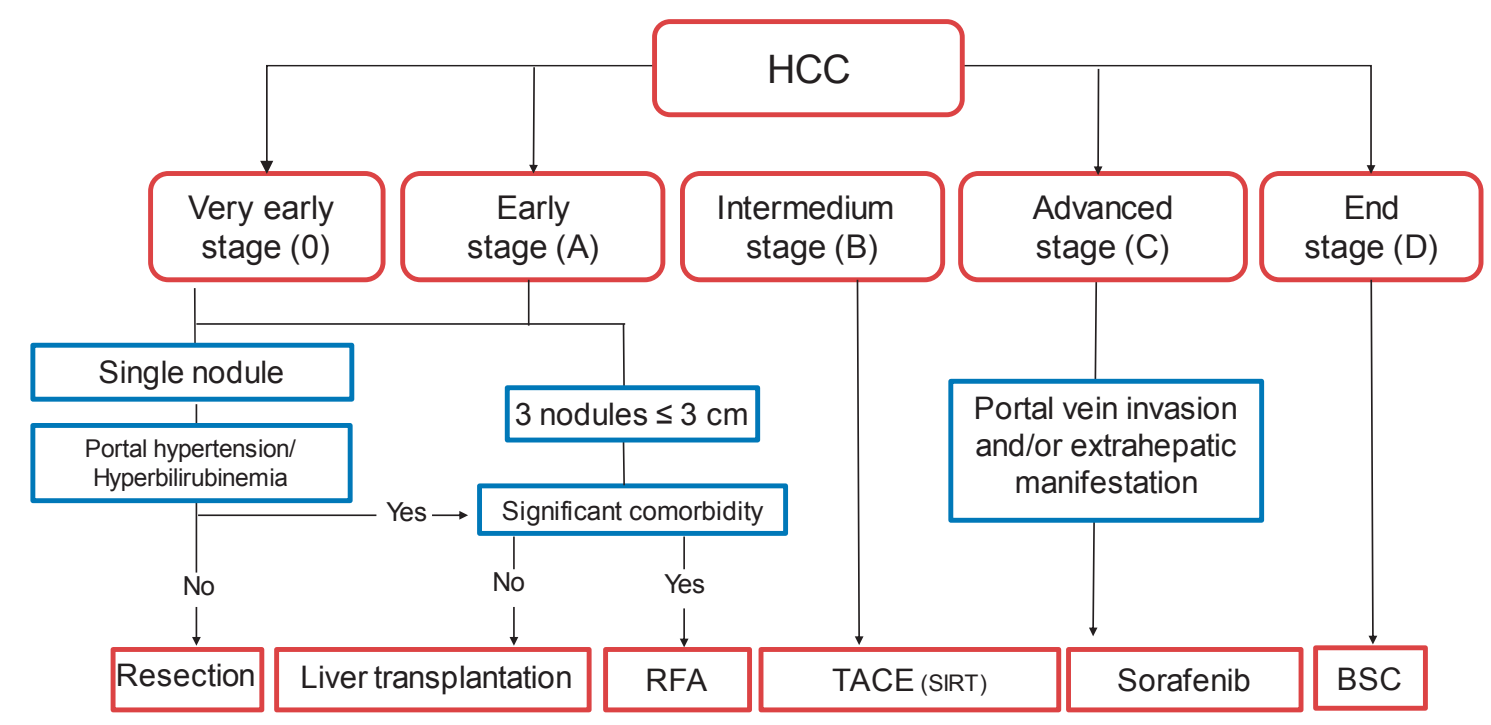

sociated with hepatobiliary procedures - is hepatic decompensation, especially in patients with a low functional hepatic reserve.

In liver cirrhosis, liver transplantation is the best therapeutic option, because removal of the entire organ erases the tumor and precancerous lesions, and the impaired liver function is restored. Early experiences with liver transplants date back to the 1980s and were mostly disappointing, with 5 -year survival rates of less than $40 \%$. After the Milan criteria were introduced, the results of liver transplantation in this indication improved notably. However, this limits organ allocation to patients with a solitary HCC nodule of less than $5 \mathrm{~cm}$ in size or 3 nodules of less than $3 \mathrm{~cm}$ in size each, and no infiltration of the portal vein or extrahepatic manifestations. By selecting patients carefully, 5-year survival rates of more than $70 \%$ can now be achieved [9]. Currently, the criteria cannot be extended to patients with larger tumors because of the shortage of organs. The Eurotransplant organ allocation criteria have been changed in December 2006, and since that time transplant waiting times for patients with HCC are much shorter. Bridging treatment such as TACE is widely used in transplant centers. Another option is living liver donation.

\section{Thermal Ablation in Early-Stage HCC}

Thermal ablation is an alternative treatment for cirrhotic patients with early-stage HCC and contraindications for surgical treatment. In this setting, procedures such as RFA or MWA are potentially curative therapeutic options. The complication rate of RFA is low, and in suitable patients with good liver function a 5 -year survival rate of up to $50 \%$ may be achieved [4]. The complications of RFA include liver abscess, biloma, and right-sided pleural effusion. Recent prospective and retrospective data showed a significant survival benefit for the combination of RFA and TACE $[10,11]$. In a randomized trial comparing TACE followed by RFA versus RFA in patients with $\mathrm{HCC}<7 \mathrm{~cm}$ in size, the combination resulted in 1-, 3-, and 4-year overall survival rates of 93, 67, and $62 \%$, respectively, compared to 85,59 , and $45 \%$ in the RFA-only arm, respectively [10]. The corresponding recurrence-free survivals were 79,61 , and $55 \%$ as well as 67,44 , and $39 \%$, respectively.

\section{Transarterial Chemoembolization}

For intermediate-stage tumors (BCLC stage B) TACE is the treatment of choice $[4,6]$. TACE is based on the simultaneous application of a chemotherapeutic agent and embolization with occluding particles. Prerequisite for successful TACE is the presence of a hypervascularized tumor. Selective administration of the mixture of chemotherapeutic agent and occluding particles results in a high local concentration of the chemotherapeutic agent in the tumor with low systemic distribution. Due to occlusion of the tumor vessels, the chemotherapeutic agents remain in the tumor region and the resulting hypoxia improves the effect of the chemotherapeutic agent. Procedures for TACE are not standardized. Commonly used chemotherapeutic agents are doxorubicin, mitomycin C, and cisplatin. Lipiodol as an oily suspension has an affinity to HCC and acts as a carrier for the chemotherapeutic agent. A favorable prognostic factor for successful TACE besides tumor size of less than $4 \mathrm{~cm}$ and increased vascularization is also uptake of lipiodol in at least $75 \%$ of the tumor volume. TACE is either performed as 'on demand' (repeated in case of persistent vascularization) or as 'continuous' (repeated every 4-6 weeks until devascularization) schedule. An alternative approach is to use doxorubicin-eluting beads for TACE. An older meta-analysis showed a survival benefit for patients with TACE [12]. Overall, 545 patients were evaluated in this study. TACE improved the 2-year survival rate with an odds ratio of 0.53 (95\% confidence interval (CI): 0.32 $0.89 ; \mathrm{p}<0.017)$ in comparison to not treated control patients. Objective response could be achieved in $35 \%$ of patients.

Since both TACE and the oral tyrosine kinase inhibitor sorafenib are active treatments for HCC, there is a rationale to combine these two treatment modalities. Liu et al. [13] performed a meta-analysis on the subject and concluded that the combination seems to be 
Fig. 3. MRI of a patient with a HCC with a typical contrast uptake in the arterial phase and $\mathbf{b}$ wash-out in the venous phase. Arrowheads indicate the tumor location.
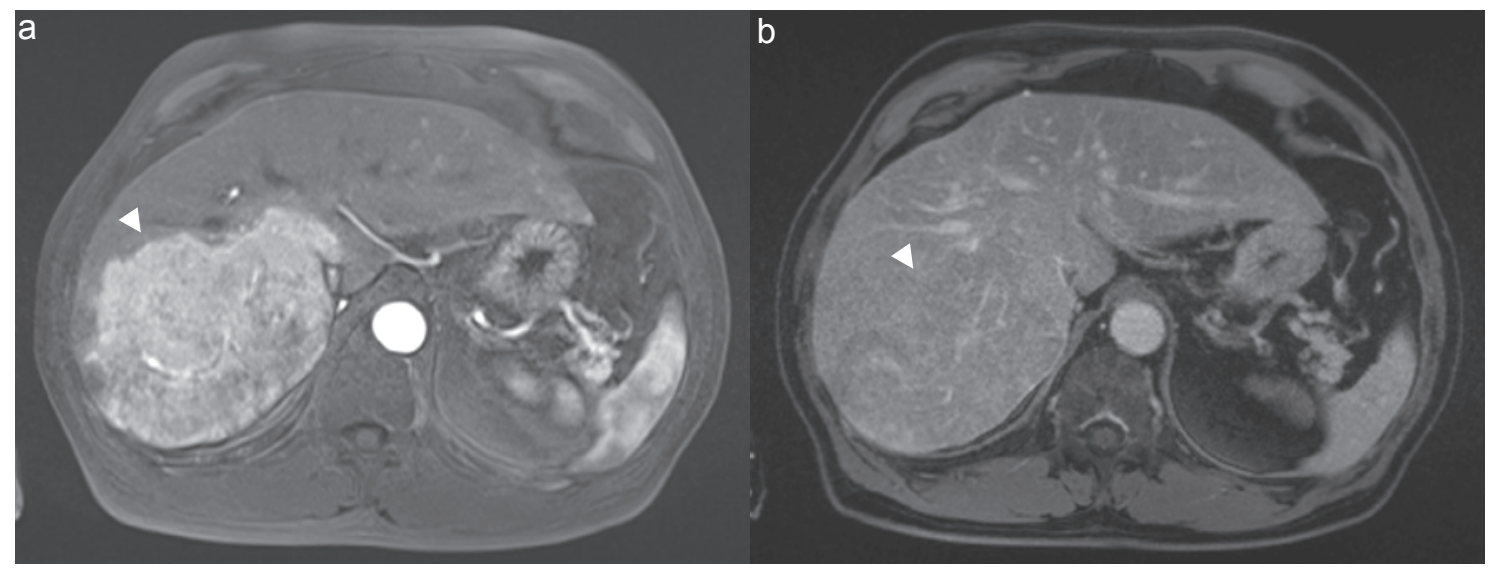

more active than each single approach. However, evidence came mainly from retrospective cohort studies. The largest reported randomized phase II study in this setting so far is the SPACE trial [14]. In this trial, the combination of TACE based on doxorubicin-eluting beads with continuous sorafenib dosing or with placebo was investigated. Although a formally positive trial, there was no meaningful progression-free survival benefit in the combination group (169 vs. 166 days in the placebo arm, hazard ratio $0.79 ; 95 \%$ CI $0.58-1.08 ; \mathrm{p}=0.072$ ). Moreover, toxicity was pronounced in the combination arm, leading to dose reductions, interruptions, and early discontinuation (e.g. nearly $50 \%$ of Western patients went offstudy during the first 3 months of treatment). Thus, hard evidence to combine TACE with sorafenib is still lacking. Toxicity of sorafenib is a limiting factor of such combination strategies.

\section{Radioembolization}

Radioembolization with yttrium-90 labelled microspheres, also known as selective intra-arterial radiotherapy (SIRT), is increasingly offered for patients with unresectable HCC who are no candidate for transplantation or ablation. Yttrium-90 labelled microspheres preferentially lodge into the tumor microvasculature if injected into the tumor-feeding vessels and lead to locally limited tumor radiation, thus minimizing radiation hazard to surrounding normal liver parenchyma. The reported median survival ranges between 7 and 42 months in cohorts of patients with different stages of HCC. However, these data are based on mainly retrospective cohort analyses, and randomized data using SIRT for HCC treatment are currently not available [15]. However, data from the randomized SORAMIC trial comparing radioembolization in combination with sorafenib versus sorafenib are awaited in the near future [16].

\section{Systemic Treatment}

Sorafenib is an oral multi-tyrosine kinase and angiogenesis inhibitor with activity against vascular endothelial growth factor receptor (VEGFR)-2, platelet-derived growth factor (PDGFR), c-Kit receptors, BRAF, and p38 signaling pathways. It is the only approved systemic treatment option in advanced-stage HCC, resulting in a relevant survival benefit compared to placebo (10.7 vs. 7.9 months) [17]. In clinical practice, these results have been confirmed by several retrospective and prospective cohort studies [18]. Underlying liver dysfunction and low tolerance of treatment toxicity may adversely affect outcomes and increase susceptibility to adverse events during treatment with sorafenib. These adverse events include fatigue (up to 40\%), diarrhea, hand-foot skin reactions, anorexia, and others. In clinical trials as well as in daily practice, they often lead to dose reduction, interruption, and early discontinuation of treatment and therefore warrant close monitoring.

Sorafenib is not eligible as adjuvant treatment for HCC. This has been recently reported in the STORM trial, which studied adjuvant treatment with sorafenib versus placebo after complete resection or ablation of HCC [19]. Sorafenib did not show an improvement in progression-free survival in this phase III trial. Therefore, adjuvant treatment with sorafenib should not be recommended in patients after complete resection or ablation.

Within the last years, several phase III trials of investigational agents in the first-line treatment of patients with advanced HCC have been reported. However, none of these drugs, e.g. sunitinib, brivanib, linifanib, or the combination of sorafenib and erlotinib, were superior to sorafenib in terms of overall survival or toxicity. Furthermore, until today there is no established systemic secondline treatment option in patients who have progressed or cannot tolerate sorafenib treatment. Currently, several agents with different targets are investigated in clinical trials in patients with advanced HCC. The most promising agents in advanced clinical development target angiogenesis (ramucirumab, lenvatinib, and regorafenib), the MET pathway (tivantinib, cabozantinib, and tepotinib), anti-PD1 antibodies (nivolumab and pembrolizumab), and oncolytic viruses (Pexa-Vec, T-Vec) [20].

\section{Disclosure Statement}

J. Trojan is serving on advisory boards for Amgen, Bayer Healthcare, Bristol Myers Squibb, Daiichi Sankyo, Lilly, and Merck Serono. 


\section{References}

1 El-Serag HB, Kanwal F: Epidemiology of hepatocellular carcinoma in the United States: where are we? Where do we go? Hepatology 2014;60:1767-1775.

2 Schurr R, Stolzel U, Schuppan D, Schwertner C, Steinberg J, Scherübl H: Zunahme des hepatozellulären und des intrahepatischen cholangiozellulären Karzinoms im Nordosten Deutschlands. Dtsch Med Wochenschr 2006;131:1649-1655.

3 Younossi ZM, Koenig AB, Abdelatif D, Fazel Y, Henry L, Wymer M: Global epidemiology of non-alcoholic fatty liver disease - meta-analytic assessment of prevalence, incidence and outcomes. Hepatology 2015;DOI: 10.1002/hep.28431

4 European Association for the Study of the Liver; European Organisation for Research and Treatment of Cancer: EASL-EORTC clinical practice guidelines: management of hepatocellular carcinoma. J Hepatol 2012;56:908-943. Erratum in: J Hepatol 2012;56:1430.

5 Zucman-Rossi J, Villanueva A, Nault JC, Llovet JM: Genetic landscape and biomarkers of hepatocellular carcinoma. Gastroenterology 2015;149:1226-1239.

6 Bruix J, Sherman M; American Association for the Study of Liver Diseases: Management of hepatocellular carcinoma: an update. Hepatology 2011;53:1020-1022.

7 Liu PH, Hsu CY, Hsia CY, Lee YH, Su CW, Huang YH, Lee FY, Lin HC, Huo TI: Prognosis of hepatocellular carcinoma: assessment of eleven staging systems. J Hepatol 2016;64:601-608.

8 Jeng WJ, Lin CC, Chen WT, Sheen IS, Lin CY, Lin SM: Adjuvant therapy for hepatocellular carcinoma after curative treatment. Dig Dis 2014;32:747-754.
Mazzaferro V, Regalia E, Doci R et al: Liver transplantation for the treatment of small hepatocellular carcinomas in patients with cirrhosis. N Engl J Med 1996; 334:693-699.

10 Xie H, Wang H, An W, Ma W, Qi R, Yang B, Liu C, Gao Y, Xu B, Wang W: The efficacy of radiofrequency ablation combined with transcatheter arterial chemoembolization for primary hepatocellular carcinoma in a cohort of 487 patients. PLoS One 2014;9:e89081.

11 Peng ZW, Zhang YJ, Chen MS, Xu L, Liang HH, Lin XJ, Guo RP, Zhang YQ, Lau WY: Radiofrequency ablation with or without transcatheter arterial chemoembolization in the treatment of hepatocellular carcinoma: a prospective randomized trial. J Clin Oncol 2013;31:426-432

12 Llovet JM, Bruix J: Systematic review of randomized trials for unresectable hepatocellular carcinoma: chemoembolization improves survival. Hepatology 2003;37: 429-442.

13 Liu L, Chen H, Wang M, Zhao Y, Cai G, Qi X, Han G: Combination therapy of sorafenib and TACE for unresectable HCC: a systematic review and meta-analysis. PLoS One 2014;9:e91124.

14 Lencioni R: Chemoembolization for hepatocellular carcinoma. Semin Oncol 2012;39:503-509.

15 Salem R, Lewandowski RJ, Kulik L, et al: Radioembolization results in longer time-to-progression and reduced toxicity compared with chemoembolization in patients with hepatocellular carcinoma. Gastroenterology 2011;140:497-507.
6 Ricke J, Bulla K, Kolligs F, Peck-Radosavljevic M, Reimer P, Sangro B, Schott E, Schütte K, Verslype C, Walecki J, Malfertheiner P; SORAMIC study group: Safety and toxicity of radioembolization plus sorafenib in advanced hepatocellular carcinoma: analysis of the European multicentre trial SORAMIC. Liver Int 2015;35: 620-626.

17 Llovet JM, Ricci S, Mazzaferro V, et al.; SHARP Investigators Study Group: Sorafenib in advanced hepatocellular carcinoma. N Engl J Med 2008;359:378-390.

18 Lencioni R, Kudo M, Ye S-L, Bronowicki J-P, Chen X-P, Dagher L, Furuse J, Geschwind JF, Ladrón de Guevara L, Papandreou C, Takayama T, Yoon SK, Nakajima K, Lehr R, Heldner S, Sanyal AJ: GIDEON (Global Investigation of therapeutic DEcisions in hepatocellular carcinoma and Of its treatment with sorafeNib): second interim analysis. Int J Clin Pract 2014;68:609-617.

19 Bruix J, Takayama T, Mazzaferro V, et al: STORM: a phase III randomized, double-blind, placebo-controlled trial of adjuvant sorafenib after resection or ablation to prevent recurrence of hepatocellular carcinoma (HCC). J Clin Oncol 2014;32(suppl):abstr 4006.

20 Waidmann O, Trojan J: Novel drugs in clinical development for hepatocellular carcinoma. Expert Opin Investig Drugs 2015;24:1075-1082. 